\title{
Aortenklappenendokarditis: Diagnostik und Therapie
}

\author{
Cornelia Piper, Lothar Faber, Dieter Horstkotte
}

Fast alle Mikroorganismen können eine Endokarditis verursachen - besonders grampositive Bakterienspezies wie Streptokokken, Enterokokken und Staphylokokken. Wenn diese im Zuge einer Bakteriämie das Endokard besiedeln, entsteht eine infektiöse Endokarditis. Erfahren Sie hier mehr über die Pathogenese, Diagnostik und Therapie dieser Erkrankung.

\section{Der konkrete Fall}

Ein 53-jähriger Mann wird wegen eines akuten zerebralen Insults mit armbetonter Hemiparese rechts und Fieber bis $39^{\circ} \mathrm{C}$ in eine neurologische Klinik aufgenommen. Er berichtet über

- rezidivierende Fieberschübe bis $39^{\circ} \mathrm{C}$,

- seit 10 Tagen neu aufgetretene Rückenschmerzen im Lendenwirbelbereich,

- Abgeschlagenheit und

- zunehmende Luftnot beim Treppensteigen.

Bekannt sind ein metabolisches Syndrom (BMI $32 \mathrm{~kg} / \mathrm{m}^{2}$ ) bei bisher nicht mit Insulin behandeltem Diabetes mellitus Typ 2 (HbA1c 9,2\%) und eine medikamentös befriedigend eingestellte arterielle Hypertonie.

Amoxicillin und Moxifloxacin | Aufgrund des unklaren Fiebers behandelte die Hausärztin den Patienten 5 Tage lang mit $2 \times 1000 \mathrm{mg}$ Amoxicillin. Nach Entfieberung trat 3 Tage nach Absetzen des Antibiotikums erneut Fieber auf. Es folgte eine zweite antibiotische Behandlung mit $1 \times 400 \mathrm{mg}$ Moxifloxacin über 5 Tage. Wieder entfieberte der Patient, fühlte sich aber schlapp und litt zunehmend unter Luftnot. 3 Tage später erlitt er einen Insult.

Aufnahmeuntersuchung I In der neurologischen Klinik fällt bei der Auskultation ein Diastolikum auf. Außerdem besteht eine Sinustachykardie mit 100 Schlägen/min. Der Blutdruck beträgt $120 / 50 \mathrm{mmHg}$. Der pulmonale und abdominelle Untersuchungsbefund ist unauffällig. Im kranialen CT zeigt sich eine kleine hypodense Raumforderung im Stromgebiet der linken A. cerebri media. Laborchemisch bestehen:

- eine Leukozytose: $14500 / \mu \mathrm{l}$

- CRP: $8 \mathrm{mg} / \mathrm{dl}$

- Fibrinogen: $480 \mathrm{mg} / \mathrm{dl}$

Transthorakale Echokardiografie I Die transthorakale Echokardiografie (TTE) zeigt eine höhergradige Aortenklappeninsuffizienz und eine kleine flottierende Auflagerung an der akoronaren Taschenklappe. Der Patient wird uns zur weiteren
Behandlung zuverlegt - es besteht der Verdacht einer Aortenklappenendokarditis mit konsekutiver zerebraler Embolie. Bei Aufnahme ist er tachykard (HF 105-138/min) und hypoton (Blutdruck $95 / 50 \mathrm{mmHg}$ ). Unter Infusion mit $4 \mu \mathrm{g} / \mathrm{min} / \mathrm{kg}$ KG Dobutamin gelingt es uns, seinen Kreislauf zu stabilisieren. Periphere Stigmata (Osler Knötchen, Janeway Effloreszenzen) finden sich nicht. In der TTE bestätigt sich der Befund einer kleinen, ca. $5 \mathrm{~mm}$ großen vegetationsverdächtigen Struktur an der akoronaren Tasche und einer höhergradigen Aortenklappeninsuffizienz bei bikuspider Klappe. Der linke Ventrikel ist enddiastolisch auf $65 \mathrm{~mm}$ dilatiert, die linksventrikuläre Ejektionsfraktion mit $50 \%$ angesichts der LV-Volumenbelastung deutlich eingeschränkt ( Abb. 1).

Transösophageale Echokardiografie | Die transösophageale Echokardiografie (TEE) zeigt, dass die Vegetation mit $12 \mathrm{~mm}$ Durchmesser erheblich grö-

Abb. 1 Transthorakale Echokardiografie (TTE): Die bikuspide Aortenklappe ist eine für eine infektiöse Endokarditis prädisponierende Klappenläsion (A: Querschnitt, B: vergrößerter Längsschnitt). An der dorsal gelegenen Tasche findet sich eine von transthorakal ca. $5 \mathrm{~mm}$ messende, flottierende, knötchenförmige Läsion (Pfeile in A und B). Die Mitralklappe erscheint per TTE nicht betroffen (Pfeilspitzen in B, D: Längsachse und in C: Vierkammerblick).

LA, RA = linker bzw. rechter Vorhof; LV, RV=linker bzw. rechter Ventrikel; Ao=Aorta

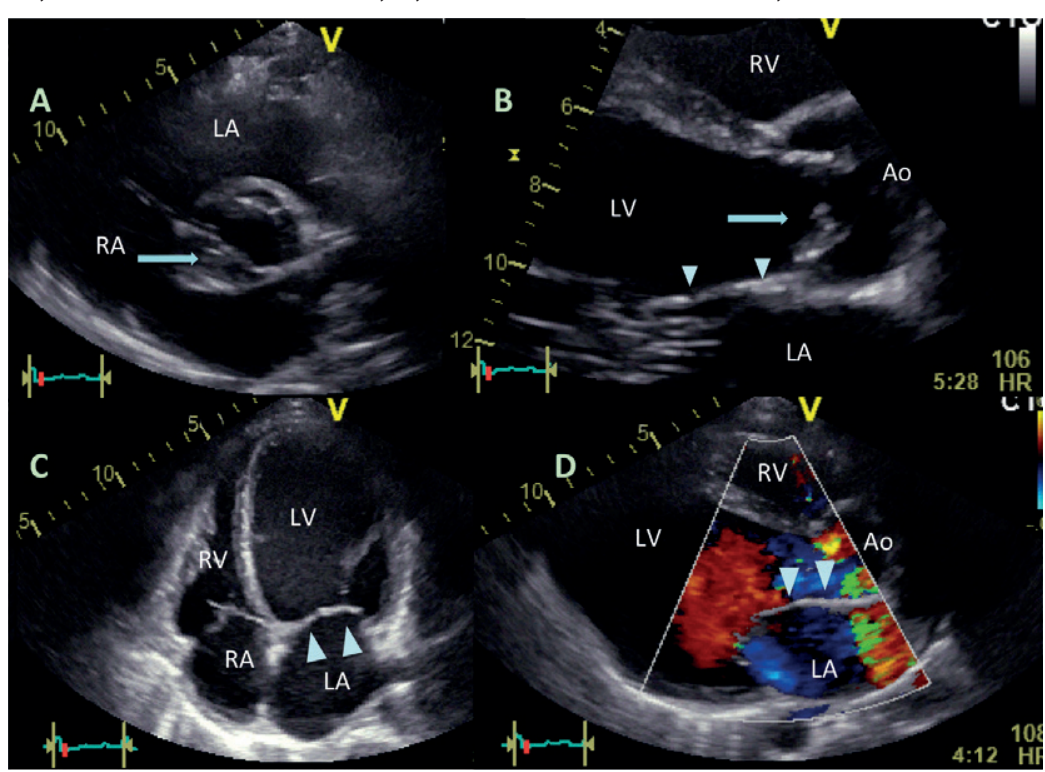




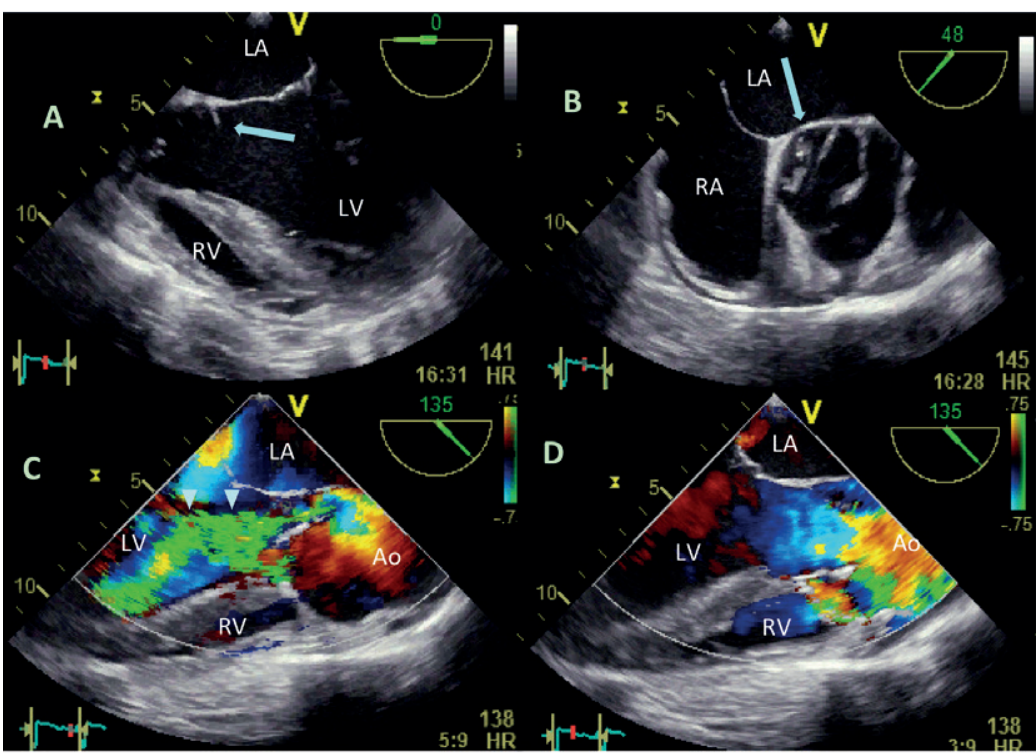

Abb. 2 Transösophageale Echokardiografie (TEE): Die Aortenklappen-Vegetation wird deutlich größer dargestellt (Pfeil in B). Man erkennt auch die kleine Abklatschvegetation im proximalen Bereich des anterioren Mitralsegels (Pfeil in A). Die Pfeilspitzen in C weisen auf den breiten, nahezu den gesamten LV-Ausflusstrakt füllenden Regurgitationsjet durch die endokarditisch destruierte Aortenklappe. D: unbehinderter antegrader Fluss durch die Klappe in der Systole.

ßer ist als mittels TTE bestimmt. Zudem weist eine der Taschenklappen eine Perforation auf. Am vorderen Mitralklappensegel können wir eine $3 \mathrm{~mm}$ große Abklatschvegetation nachweisen ( $\bullet$ Abb. 2).

Antiobiotikatherapie | Die präoperative Koronarangiografie schließt eine koronare Herzkrankheit aus. Nach Abnahme von $3 \times 2$ Blutkulturen im Abstand von je einer Stunde beginnen wir nach zwei Stunden mit einer kalkulierten antibiotischen Behandlung: $2 \times 1500 \mathrm{mg}$ Vancomycin und $3 \times 100 \mathrm{mg}$ Gentamicin intravenös, da bei Diabetes mellitus häufig auftretende Erreger wie Staphylokokken und Enterokokken bei der Wahl der Antibiotika zu berücksichtigen sind. Angesichts des destruierenden Verlaufs der Endokarditis ist eine gramnegative Endokarditis unwahrscheinlich. Deshalb erhält der Patient kein Ciprofloxacin - obwohl dies die Leitlinien bei unklaren Endokarditisfällen und Betalactamunverträglichkeit vorsehen.

Aortenklappenersatz | Nachdem wir mittels erneuter kranialer CT-Untersuchung eine zerebrale Reperfusionsblutung ausschließen konnten, wird der Patient notfallmäßig operiert.

Ein Aortenklappenersatz ist schnell vorzunehmen: Der Patient ist bei hochgradiger Aortenklappeninsuffizienz hämodynamisch instabil und katecholaminpflichtig.

Zudem hat er aufgrund der $42 \mathrm{~h}$ zuvor stattgehabten zerebralen Embolie und einer nach wie vor großen Vegetation ( $>10 \mathrm{~mm}$ ) an der Aortenklappe ein deutlich erhöhtes Risiko für eine erneute thromboembolische Komplikation. Intraoperativ zeigt sich zudem ein Aortenwurzelabszess - dieser war auch bei retrospektiver Nachbefundung im präoperativen TEE nicht erkennbar. Zur Sanierung der Endokarditis müssen neben der Aortenklappe Teile der Aortenwurzel reseziert werden. Nach Aortenwurzelrekonstruktion wird eine mechanische Doppelflügelprothese (SJM A $29 \mathrm{~mm}$ ) implantiert. Die Abklatschvegetation am vorderen Mitralklappensegel kann mechanisch entfernt werden.

Weiterer Verlauf I Der Keim (Staphylococcus aureus) erweist sich als oxacillinempfindlich $\left(\mathrm{MHK}_{\mathrm{Oxa}}<1 \mathrm{mg} / \mathrm{L}\right)$, sodass wir die intravenöse Antibiotikatherapie auf $3 \times 4 \mathrm{~g}$ Oxacillin und $3 \times 1 \mathrm{mg} / \mathrm{kg}$ KG Gentamicin umstellen. Gentamicin verabreichen wir über 3 Tage und Oxacillin über 4 Wochen intravenös. 5 Tage nach dem Aortenklappenersatz entwickelt der Patient einen AV-Block III. Grades. Dadurch wird eine Vorhofund Ventrikelstimulation über die epikardialen Schrittmacherdrähte erforderlich, die intraoperativ bei Aortenklappenendokarditis mit Abszess routinemäßig angelegt werden. Der Mann entfiebert, Leukozyten und CRP normalisieren sich. Auch die linksventrikuläre Dilatation ist rückläufig und die linksventrikuläre Funktion verbessert sich. Eine Woche nach Ende der antibiotischen Therapie ist der Patient anhaltend fieberfrei und der CRP-Wert liegt weiterhin im Normbereich, sodass bei fortbestehendem AV-Block III. Grades jetzt ein DDD-Schrittmacher implantiert wird. Erfreulicherweise hat sich zwischenzeitlich die rechtsseitige Hemiparese bis auf eine Sensibilitätsstörung des rechten Arms zurückgebildet.

\section{Pathogenese und Prädisposition}

Nahezu alle Mikroorganismen können eine Endokarditis verursachen. Jedoch dominieren grampositive Bakterienspezies wie Streptokokken, Enterokokken und Staphylokokken. Voraussetzung für die Entstehung einer infektiösen Endokarditis (IE) ist die Besiedlung des Endokards durch vermehrungsfähige Mikroorganismen im Zuge einer Bakteriämie. Prädisponierende Faktoren sind

- Diabetes mellitus (Hautläsionen, diabetisches Fußsyndrom),

- terminale Niereninsuffizienz (Dialyse),

- Leberzirrhose,

- Virushepatitis

- Alkoholabusus,

- immunsuppressive Therapie,

- Bestrahlung,

- angeborene und erworbene Immundefekte,

- Malignome,

- Drogenabusus (intravenöse Injektionen),

- Verbrennungen,

- Polytraumata (erhöhte Bakteriämiefrequenzen) und

- Klappenveränderungen (wie bikuspide Aortenklappe) $[1,2]$. 


\section{Allgemeinsymptomatik}

Allgemeine Krankheitssymptome wie

- Abgeschlagenheit,

- Mattigkeit,

- rezidivierende Schweißausbrüche und

- Leistungsknick

bestehen bei nahezu allen Patienten. Etwa 90\%

der Patienten weisen kontinuierliches oder remittierendes Fieber auf.

Fehlen kann das Fieber bei älteren Patienten mit subakuten Verlaufsformen, bei Patienten mit terminaler Herzinsuffizienz, zerebralen Blutungen oder medikamentenbedingt.

Ein neu aufgetretenes Geräusch infolge der Herzklappeninsuffizienz ist diagnostisch verwertbar man muss es jedoch gegen systolische Geräusche bei Patienten mit akuten (erhöhtes Herzminutenvolumen) oder chronischen Infekten (Anämie) abgrenzen.

Dringender Verdacht | Folgende Konstellationen legen den dringenden Verdacht auf eine Endokarditis nahe [3]:

- Fieber plus

- intrakardiales Polymermaterial

> andere Hochrisiko-Prädisposition

- neue Arrhythmien oder Überleitungsstörungen

- Erstmanifestation einer Herzinsuffizienz

> positive Blutkultur (mit „typischen“ Endokarditiserregern)

- Haut- und Retinamanifestationen

- multifokale/wechselnde Lungeninfiltrate

- periphere Abszesse ungeklärter Ätiologie

> Prädisposition und stattgehabte Interventionen/Diagnostik (Bakteriämie)

- „neuer“ Klappenfehler/(Insuffizienz-) Geräusch

- Embolien ungeklärter Ätiologie

- Sepsis ungeklärter Ätiologie

- Hämaturie, Glomerulonephritis, V.a. Niereninfarkt

\section{Manifestationen an Haut und Augen}

Klassische Haut- und Augenmanifestationen sind:

- Osler-Knötchen: druckschmerzhafte, stecknadelkopf- bis erbsengroße, blau-rote oder bläuliche Schwellungen, meist an den Finger- und Zehenkuppen; sie sind Folge peripherer Mikroembolien und einer konsekutiven Vaskulitis

- Janeway-Effloreszenzen: schmerzlose, unter Druck abblassende, makulöse, 1-5 mm große, unregelmäßig begrenzte, hämorrhagische Effloreszenzen an Handflächen und Fußsohlen, gelegentlich auch an Armen, Beinen und Bauch

- Roth-Flecken in der Retina: Sie zeigen sich als Cotton-Wool-Herde, denen perivasale Lymphozytenaggregate, Ödeme und Blutungen zugrunde liegen.
Daneben finden sich häufig subunguale Blutungen (Splinter-Blutungen) und Petechien, die jedoch unspezifisch sind.

Krankheitsverlauf | Während der letzten 30 Jahre hat sich das klinische Erscheinungsbild der Endokarditis verändert: Die Zahl von StreptokokkenEndokarditiden war rückläufig, gleichzeitig haben Staphylokokken und Enterokokken als Endokarditiserreger zugenommen. Dies hat wesentlich zum Anstieg akuter, zum Teil foudroyanter Krankheitsverläufe beigetragen [2, 3-5].

\section{Anamnese}

Allgemein | Bei der Anamnese fragt man gezielt nach

- der Dauer und dem Verlauf von Fieber,

- Unwohlsein,

- Blässe,

- Leistungsminderung,

- Luftnot

- Arthralgien und

- Hautveränderungen.

Aus den Antworten lässt sich abschätzen, seit wann die Infektionssymptomatik besteht und ob es bereits Hinweise für die Entwicklung einer Herzinsuffizienz auf dem Boden zunehmender Herzklappendestruktionen gibt.

Speziell | Die spezielle Anamnese dient der Erfragung vorbekannter Herzgeräusche, zur Endokarditis prädisponierender Herzfehler und von Umständen, die mit einer Bakteriämie einhergegangen sein könnten [1-3, 6].

\section{Laborbefunde}

Leukozytose, BSG- und CRP-Erhöhungen gehen regelhaft mit einer akuten IE einher, sind wegen den vielen möglichen Ursachen aber von geringer differenzialdiagnostischer Bedeutung. Bei subakuten Krankheitsverläufen kann eine Leukozytose fehlen. Leukopenien können durch gramnegative Erreger oder eine antibiotische Therapie bedingt sein. Allerdings schließt ein normales CRP eine Endokarditis praktisch aus. Bei Verdacht auf Sepsis ist der Procalcitoninspiegel hilfreich [1, 3-5].

\section{Echokardiografie}

Besteht ein dringlicher Verdacht auf eine Endokarditis, muss man leitliniengerecht unverzüglich eine transthorakale Echokardiografie (TTE) vornehmen [3, 7]. Eine transösophageale Echokardiografie (TEE) ist unverzichtbar bei

- möglicher Beteiligung intrakardialer Implantate (Herzklappenprothesen, Schrittmacher, Defibrillatoren, etc.),

- unzureichender Bildqualität der TTE, 


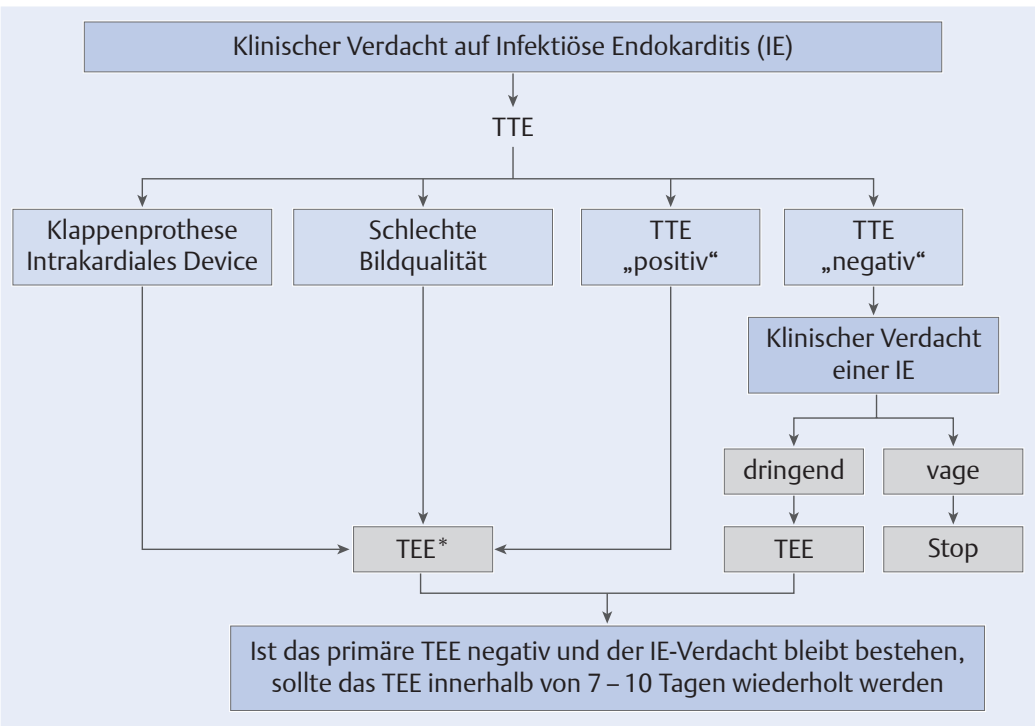

Abb. 3 Indikationen zur transthorakalen und transösophagealen Echokardiografie bei vermuteter IE (gemäß [3]).

$\mathrm{IE}=$ infektiöse Endokarditis; TEE = transösophageale Echokardiografie; TTE = transthorakale Echokardiografie

\section{Mikrobiologische Befunde}

Obligate Bestandteile der mikrobiologischen Diagnostik sind

- die Erregeridentifikation bis zur Spezies und

- die Testung der minimalen inhibitorischen Konzentration (MHK) der Antibiotika im quantitativen Reihenverdünnungstest.

Eine antimikrobielle Therapie aufgrund des Ergebnisses im Agardiffusionstest geht mit erhöhter Morbidität und Mortalität einher und ist nicht leitliniengerecht [3].

Erregerspezies | Grampositive Erregerspezies wie Streptokokken, Staphylokokken und Enterokokken dominieren, weil sie besonders gut an endokardständigen Thromben haften. Staphylokokkus epidermidis verursachen häufig Polymer-assoziierte Endokarditiden (Elektroden, Klappenprothesen), da ein Teil dieser Erreger irreversibel an Polymeroberflächen haften und eine Matrix bilden kann [4]. Weitere Verursacher sind grampositive und gramnegative Stäbchenbakterien, insbesondere

- unbestätigtem Endokarditisverdacht oder

- Notwendigkeit einer chirurgischen Intervention während florider IE [1, 3, 8-11] ( Abb. 3).

\section{Blutkulturen}

Abnahme und Lagerung I Den Leitlinien entsprechend soll man - unabhängig vom Fieberverlauf - mindestens drei Blutkulturen jeweils aerob und anaerob zu unterschiedlichen Zeitpunkten aus peripheren Venen abnehmen [3]. Arteriell gewonnene Blutkulturen sind venösen unterlegen. Blutkulturen, die in Fieberspitzen abgenommen wurden, verringern die Erfolgsaussichten [1]. Die Proben sollen den Mikrobiologen rasch erreichen müssen sie zwischengelagert werden, dürfen sie keiner übermäßigen Wärme (Brutschrank) oder Kälte (Kühlschrank) ausgesetzt werden. Vielmehr soll man sie bei Zimmertemperatur lagern. Ist ein rascher Therapiebeginn zwingend, ist es sinnvoll, vor Einleitung der antimikrobiellen Therapie drei Blutkulturpaare im Abstand von je einer Stunde abzunehmen.

Kulturnegative Endokarditiden | Der nach wie vor hohe Anteil kulturnegativer Endokarditiden (KNE) belegt diagnostische Mängel: Bei einem Teil der Patienten bleiben positive Kulturen aus, da ungezielt antibiotische Therapien begonnen wurden. Diese sind prognostisch ungünstig und sollten bei allen nicht kritisch kranken Patienten mit KNE abgesetzt werden. Nach Abklingen der antibiotischen Wirkung (3-7 Tage) sind die Erfolgsaussichten für einen Erregernachweis höher. Bei KNE stehen moderne PCR-Methoden zur Verfügung [12, 13]. Eingesetzt werden die 23S rDNA real-time PCR oder16S rDNA PCR für Bakterien, sowie 28S rDNA PCR für Pilze [12].
- solche der HACEK-Gruppe (Haemophilus, Actinobazillus, Cardiobacterium hominis, Eikenella corrodens, Kingella kingae),

- gramnegative Kokken,

- Mykobakterien,

- Rikettsien,

- Chlamydien und

- Anaerobier (insbesondere Peptostreptokokken).

Endokarditiden infolge einer Infektion mit Bartonellen, Brucellen und Coxiella spp. lassen sich nur mit serologischen Untersuchungen sichern [14].

Der Streptococcus bovis Biotyp I ist regelhaft mit gastrointestinalen Tumoren assoziiert. Bei diesen Patienten ist rasch eine Gastro- und Koloskopie durchzuführen [6].

\section{Allgemeine Therapieprinzipien}

Flüssigkeit und Elektrolyte I Die konservative Therapie umfasst den Ausgleich der Flüssigkeitsund Elektrolytbilanz. Auf Verweilkatheter sollte man möglichst verzichten - andernfalls sollten sie alle 8 Tage gewechselt werden.

Gezielte Sanierung I Während der Antibiotikatherapie soll eine kausale Infektionsquelle (Erregerübereinstimmung) gezielt saniert werden. Eine ungezielte Elimination möglicher Infektionsquellen ist nicht sinnvoll. Die früher gefürchteten endogenen Rezidivinfektionen - d.h. neuerliche Infektionen aus einer Bakteriämiequelle, die bereits die ursprüngliche IE verursachte - ist klinisch bedeutungslos: Ihre Inzidenz ist mit und ohne systematische Sanierung gleichermaßen niedrig (unter 1\%). 
Therapiedauer I Eine unzureichende Therapiedauer erhöht die Gefahr von Rezidivinfektion.

Man soll deshalb auch bei unkomplizierten Krankheitsverläufen eine 4-wöchige intravenöse Therapie im Regelfall nicht unterschreiten.

Bei Prothesenendokarditiden sind meist 6-wöchige Therapien erforderlich. Ist zur Behandlung einer floriden Endokarditis ein dringlicher Herzklappenersatz erforderlich, zählt der Operationstag als Tag 1 der Antibiotikabehandlung [3].

Antikoagulation | Antikoagulanzien und Heparin haben keinen Einfluss auf die Inzidenz thromboembolischer Komplikationen. Klinisch und tierexperimentell gehen sie jedoch mit einer erhöhten Rate von Blutungskomplikationen einher [1, 3].

\section{Antibiotische Therapie}

Parenterale Applikation | Bei fehlender Vaskularisierung des Endokards und der Vegetation ist ein hoher Diffusionsgradient des Antibiotikums bzw. der Antibiotikakombination notwendig, um den Expositionsschutz zu überwinden. Es ist deshalb unerlässlich, bakterizid wirksame Antibiotika auszuwählen und hohe Serumspiegel zu erreichen. Dies ist nur mit parenteraler Gabe möglich. Eine Übersicht über die antibiotischen Therapieschemata gibt $>$ Tab. 1 .

Keim identifizieren | Ist der Patient nicht kritisch krank, soll immer versucht werden, den Keim vor der antibiotischen Therapie zu identifizieren. In zahlreichen Fällen haben wir bei fehlendem Erregernachweis auswärts begonnene antibiotische Behandlungen bis zu einer Woche pausiert, um danach den Keim aus Blutkulturen zu isolieren und den Patienten gezielt antibiotisch zu behandeln. Gelingt der Keimnachweis nicht oder ist eine unverzügliche Behandlung mit Antibiotika erforderlich, ist ein Spezialist für Infektionskrankheiten zu kontaktieren - die Datenlage zu den in den Leitlinien empfohlenen Antibiotika-Regimen hat einen niedrigen Evidenzgrad von IIbC [3].

Monitoring der antibiotischen Therapie | Während einer Endokarditis-Therapie ist es besonders wichtig, die Wirkspiegel zu kontrollieren: Die renalen und hepatischen Exkretionsmechanismen werden auch durch die Begleitmedikation häufig gestört. Bei IE ist die Nierenfunktion in mehr als der Hälfte der Fälle beeinträchtet. VancomycinTalspiegel sollen bei $10-15 \mathrm{mg} / \mathrm{l}$ und GentamicinTalspiegel unter $1 \mathrm{mg} / 1$ liegen.

Penicillinsensible Streptokokken | Die Standardtherapie besteht in der Kombination von Penicillin G und einem Aminoglykosid - eine synergistische Wirkung beider Substanzen wird meist selbst dann erzielt, wenn der Erreger gegen Aminoglykoside allein wenig empfindlich ist.

Enterokokken I In den letzten Jahren haben Enterokokken (insbesondere E. faecalis) eine Toleranz gegen zahlreiche zellwandaktive Antibiotika ( $\beta$-Laktam-Antibiotika und Vancomycin) erworben. Die MHK und die minimale bakterizide Konzentration (MBK) unterscheiden sich meist um mehrere Titerstufen. Hohe Penicillindosen verschlechtern die Bakterizidie oft (Eagle-Effekt).

Eine synergistisch wirksame Kombination z. B. von Penicillin mit einem Aminoglykosid potenziert die bakterizide Wirkung und verbessert die Heilungsaussichten.

Für die Auswahl des Aminoglykosids ist wesentlich, dass bei bis zu 80\% der E.-faecalis-Stämme eine „High Level Resistance (HLR)“ gegen Streptomycin besteht. Eine HLR gegenüber Gentamicin liegt in Deutschland dagegen bislang nur selten vor. Bei E.-faecium-Stämmen ist die Therapie mit Gentamicin nicht sinnvoll, da deren AminoglykosidAzetyltransferase auch Gentamicin inaktiviert.

In jedem Fall muss man die synergistische Wirksamkeit verschiedener Aminoglykoside mikrobiologisch prüfen. In Kombination mit Gentamicin können prinzipiell Vancomycin oder Ampicillinderivate eingesetzt werden. Tierexperimentell und in vitro besitzt das Ampicillinderivat Mezlocillin die günstigste Aktivität. Die 4-wöchige Kombination von Amoxicillin oder Ampicillin und Gentamicin gilt als Therapie der Wahl. Bei komplizierten Verläufen ist eine 6-wöchige Therapie zu empfehlen.

Für Patienten mit Penicillinunverträglichkeit sind Vancomycin, in Einzelfällen und in beschränktem Umfang auch Daptomycin und Linezolid (cave Toxizität) erprobt $[15,16]$. Bei vanA/vanB-Resistenzen (vanA, vanB=Vancomycinresistenz-Gene) wurde in Einzelfällen mit Erfolg Imipenem plus Ampicillin oder Ceftriaxon plus Ampicillin eingesetzt $[17,18]$.

Staphylokokken I Mehr als 80\% der Staphylokokken produzieren Penicillin- $\beta$-Laktamase (Penicillinresistenz). Staphylococcus-aureus-Stämme sind jedoch sensibel auf Isoxazolylpenicilline und Cephalosporine $\left(\mathrm{MHK}_{\mathrm{Oxa}}<1 \mathrm{mg} / \mathrm{L}\right)$. In Tierexperimenten resultiert die Kombination mit einem Aminoglykosid in einer rascheren Sterilisierung der Vegetation und einer günstigen Prognose. Ist die Nephrotoxizität deutlich erhöht und der Überlebensvorteil unsicher, empfiehlt man die zusätzliche Therapie einer nativen Herzklappen-IE mit Gentamicin nur noch optional für 3 Tage - bei Polymer-assoziierter IE jedoch für 14 Tage [3]. Bei der Antibiotikawahl muss man zudem berücksichtigen, dass die Raten an oxacillinresistenten 


\begin{tabular}{|c|c|c|c|c|c|}
\hline Erreger & sonstige Bedingungen & Antibiotika, Antimykotika & Dosierung & Therapiedauer & Evidenzgrad \\
\hline \multirow[t]{4}{*}{$\begin{array}{l}\text { Penicillin-empfindliche } \\
\text { Streptokokken } \\
\left(\mathrm{MHK}_{\mathrm{Pen}}<0,125 \mathrm{mg} / \mathrm{L}\right)\end{array}$} & \multirow[t]{2}{*}{ Penicillinverträglichkeit } & $\begin{array}{l}\text { Penicillin } \mathrm{G}^{\mathrm{a}, \mathrm{b}} \text { oder } \\
\text { Ampicillin }\end{array}$ & $\begin{array}{l}12-18 \mathrm{Mio} \text { E } / 24 \mathrm{~h} \text { in } 6 \text { ED } \\
100-200 \mathrm{mg} / \mathrm{kg} / 24 \mathrm{~h} \text { in } \\
\text { 4-6 Dosen }\end{array}$ & $\begin{array}{l}\text { mindestens } 4 \\
\text { Wochen }^{\mathrm{c}, \mathrm{o}}\end{array}$ & \multirow[t]{2}{*}{ IB } \\
\hline & & $\begin{array}{l}\text { nur bei verkürzter } \\
\text { Therapie: plus } \\
\text { Gentamicin } \\
\text { c,d,e }\end{array}$ & $3 \mathrm{mg} / \mathrm{kg} / 24 \mathrm{~h}$ als ED & 2 Wochen & \\
\hline & \multirow{2}{*}{$\begin{array}{l}\text { Penicillinunverträglich- } \\
\text { keit und / oder Nierenin- } \\
\text { suffizienz (nicht } \\
\text { stationäre Patienten) } \\
\text { Penicillin- und Cephalo- } \\
\text { sporinunverträglichkdeit }\end{array}$} & $\begin{array}{l}\text { Ceftriaxon } \\
\text { nur bei verkürzter Therapie: } \\
\text { plus } \\
\text { Gentamicin }{ }^{\mathrm{c}, \mathrm{d}, \mathrm{e}}\end{array}$ & $2 \mathrm{~g} / 24 \mathrm{~h}$ als ED & 4 Wochen ${ }^{\mathrm{c}, 0}$ & IB \\
\hline & & Vancomycin $^{f}$ & 2 -mal $15 \mathrm{mg} / \mathrm{kg} / 24 \mathrm{~h}$ & 4 Wochen $^{\circ}$ & IC \\
\hline \multirow[t]{3}{*}{$\begin{array}{l}\text { mäßig empfindliche } \\
\text { Streptokokken } \\
\left(\mathrm{MHK}_{\mathrm{Pen}} 0,125-2 \mathrm{mg} / \mathrm{L}\right)\end{array}$} & \multirow[t]{2}{*}{ Penicillinverträglichkeit } & $\begin{array}{l}\text { Penicillin G oder } \\
\text { Ampicillin } \\
\text { plus }\end{array}$ & $\begin{array}{l}24 \mathrm{Mio} \text { E } / 24 \mathrm{~h} \text { in } 6 \mathrm{ED} \\
200 \mathrm{mg} / \mathrm{kg} / 24 \mathrm{~h} \text { in } 4-6 \mathrm{ED}\end{array}$ & $\begin{array}{l}4 \text { Wochen }^{\circ} \\
4 \text { Wochen }^{\circ}\end{array}$ & \multirow[t]{2}{*}{ IB } \\
\hline & & Gentamicin $^{\mathrm{d}}$ & $3 \mathrm{mg} / \mathrm{kg} / 24 \mathrm{~h}$ als ED & 2 Wochen & \\
\hline & $\begin{array}{l}\text { Penicillinunverträglich- } \\
\text { keit }\end{array}$ & $\begin{array}{l}\text { Vancomycin }{ }^{f} \text { plus } \\
\text { Gentamicin }^{d}\end{array}$ & $\begin{array}{l}2-\mathrm{mal} 15 \mathrm{mg} / \mathrm{kg} / 24 \mathrm{~h} \\
3 \mathrm{mg} / \mathrm{kg} / 24 \mathrm{~h} \text { als ED }\end{array}$ & 4 Wochen $^{\circ}$ & IC \\
\hline $\begin{array}{l}\text { Enterokokken und } \\
\text { Penicillin-resistente } \\
\text { Streptokokken }\end{array}$ & Penicillinverträglichkeit & $\begin{array}{l}\text { Ampicillin oder } \\
\text { Penicillin G plus } \\
\text { Gentamicin }^{\mathrm{d}, \mathrm{i}}\end{array}$ & $\begin{array}{l}200 \mathrm{mg} / \mathrm{kg} / 24 \mathrm{~h} \text { in } 4-6 \text { ED } \\
24 \text { Mio E } / 24 \mathrm{~h} \text { in } 6 \text { ED } \\
3-\mathrm{mal} 1 \mathrm{mg} / \mathrm{kg} / 24 \mathrm{~h}\end{array}$ & $\begin{array}{l}\text { 4-6 Wochen } \\
\text { 4-6 Wochen }{ }^{i} \\
\text { 4-6 Wochen }{ }^{i}\end{array}$ & IB \\
\hline $\begin{array}{l}\left(\mathrm{MHK}_{\text {Pen }} 2-8 \mathrm{mg} / \mathrm{L}\right. \\
\left.\text { MHK }_{\text {Genta }}<500 \mathrm{mg} / \mathrm{L}\right)\end{array}$ & $\begin{array}{l}\text { Penicillinunverträglich- } \\
\text { keit }\end{array}$ & $\begin{array}{l}\text { Vancomycin }{ }^{\mathrm{f}, \mathrm{i}} \text { plus } \\
\text { Gentamicin }^{\mathrm{d}, \mathrm{g}, \mathrm{i}}\end{array}$ & $\begin{array}{l}2-\mathrm{mal} 15 \mathrm{mg} / \mathrm{kg} / 24 \mathrm{~h} \\
3-\mathrm{mal} 1 \mathrm{mg} / \mathrm{kg} / 24 \mathrm{~h}\end{array}$ & $\begin{array}{l}6 \text { Wochen }^{\mathrm{i}, \mathrm{k}} \\
6 \text { Wochen }^{\mathrm{i}, \mathrm{k}}\end{array}$ & IC \\
\hline \multirow[t]{2}{*}{$\begin{array}{l}\text { Oxacillin-empfindliche } \\
\text { Staphylokokken (MSSA) } \\
\left(\mathrm{MHK}_{\text {0xa }}<1 \mathrm{mg} / \mathrm{L}\right)\end{array}$} & Penicillinverträglichkeit & $\begin{array}{l}\text { Di- oder } \\
\text { Flucloxacillin }{ }^{\mathrm{a}} \text { plus } \\
\text { Gentamicin } \\
\text { d,g,m }\end{array}$ & $12 \mathrm{~g} / 24 \mathrm{~h}$ in $4-6 \mathrm{ED}$ & 4-6 Wochen ${ }^{k, o}$ & IB \\
\hline & $\begin{array}{l}\text { Penicillinunverträglich- } \\
\text { keit }\end{array}$ & $\begin{array}{l}\text { Vancomycin }{ }^{\mathrm{f}, \mathrm{p}} \text { plus } \\
\text { Gentamicin }^{\mathrm{d}, \mathrm{g,m}}\end{array}$ & $\begin{array}{l}2-\mathrm{mal} 15 \mathrm{mg} / \mathrm{kg} / 24 \mathrm{~h} \\
3-\mathrm{mal} 1 \mathrm{mg} / \mathrm{kg} / 24 \mathrm{~h}\end{array}$ & $\begin{array}{l}\text { 4-6 Wochen }{ }^{k, o} \\
\text { 3-5 Tage }\end{array}$ & IB \\
\hline $\begin{array}{l}\text { Oxacillin-resistente } \\
\text { Staphylokokken } \\
\left(\mathrm{MHK}_{\text {Oxa }}>1 \mathrm{mg} / \mathrm{L}\right)\end{array}$ & \multirow[t]{3}{*}{$\begin{array}{l}\text { Empfindlichkeitsprüfung } \\
\text { in vitro }\end{array}$} & $\begin{array}{l}\text { Vancomycin }{ }^{\mathrm{f}, \mathrm{m}, \mathrm{p}} \text { plus } \\
\text { Gentamicin }^{\mathrm{d}, \mathrm{g,m}}\end{array}$ & $\begin{array}{l}2-\mathrm{mal} 15 \mathrm{mg} / \mathrm{kg} / 24 \mathrm{~h} \\
3-\mathrm{mal} 1 \mathrm{mg} / \mathrm{kg} / 24 \mathrm{~h}\end{array}$ & $\begin{array}{l}6 \text { Wochen } \\
\text { 3-5 Tage, } \\
2 \text { Wochen bei PVE }\end{array}$ & \multirow[t]{2}{*}{ IB } \\
\hline $\begin{array}{l}\text { Pseudomonas } \\
\text { aeruginosa }\end{array}$ & & $\begin{array}{l}\text { Piperacillin }{ }^{a} \text { plus } \\
\text { Tobramycin }^{d}\end{array}$ & $\begin{array}{l}3-\mathrm{mal} 100 \mathrm{mg} / \mathrm{kg} / 24 \mathrm{~h} \\
3 \mathrm{mg} / \mathrm{kg} / 24 \mathrm{~h} \text { in } 2-3 \mathrm{ED}\end{array}$ & $\begin{array}{l}\text { mind. } 6 \text { Wochen } \\
\text { mind. } 6 \text { Wochen }\end{array}$ & \\
\hline $\begin{array}{l}\text { E. coli, Klebsiellen, } \\
\text { Serratia, Proteus, } \\
\text { Enterobakter }\end{array}$ & & $\begin{array}{l}\text { Cefotaxim plus } \\
\text { Gentamicin }\end{array}$ & $\begin{array}{l}4-\mathrm{mal} 2 \mathrm{~g} / 24 \mathrm{~h} \\
3 \mathrm{mg} / \mathrm{kg} / 24 \mathrm{~h} \text { in } 2-3 \mathrm{ED}\end{array}$ & $\begin{array}{l}\text { 4-6 Wochenk } \\
\text { 4-6 Wochenk }\end{array}$ & \\
\hline $\begin{array}{l}\text { Hämophilus, Actinoba- } \\
\text { cillus, Cardiobacterium } \\
\text { hominis, Eikenella, } \\
\text { Kingella (HACEK) }\end{array}$ & Penicillinempfindlichkeit & $\begin{array}{l}\text { Ceftriaxon } \\
\text { Ampicillin plus } \\
\text { Gentamicin }^{d}\end{array}$ & $\begin{array}{l}2 \mathrm{~g} / 24 \mathrm{~h} \text { in einer ED } \\
12 \mathrm{~g} / 24 \mathrm{~h} \text { in } 3-4 \mathrm{ED} \\
3 \mathrm{mg} / \mathrm{kg} / 24 \mathrm{~h} \text { in } 2-3 \mathrm{ED}\end{array}$ & $\begin{array}{l}\text { 4 Wochen } \\
\text { 4-6 Wochen } \\
\text { 4-6 Wochen }\end{array}$ & \\
\hline Candida und andere & & Amphotericin B plus & $1 \mathrm{mg} / \mathrm{kg} / 24 \mathrm{~h}$ 3-bis 4-mal & 6-8 Wochen & \\
\hline Pilze & & $\begin{array}{l}\text { Flucytosin } \\
\text { Caspofungin }\end{array}$ & $\begin{array}{l}50 \mathrm{mg} / \mathrm{kg} / 24 \mathrm{~h} \\
\text { 1. Tag } 70 \mathrm{mg} \text { als ED, ab } 2 . \\
\text { Tag } 50 \mathrm{mg} \text { als ED (> } 80 \mathrm{~kg} \\
\text { KG } 70 \mathrm{mg} \text { als ED) }\end{array}$ & 6-8 Wochen & \\
\hline
\end{tabular}

Tab. 1 Empfehlungen zur Antibiotikatherapie bei infektiösen Endokarditiden (nach [4]).

Fußnoten s. nächste Seite
S.-aureus- und S.-epidermidis-Stämmen zunehmen. Hier bietet sich neben Vancomycin nach Rücksprache mit einem Kompetenzzentrum der Einsatz von hochdosiertem Daptomycin an $(1 \times 8-12 \mathrm{mg} / \mathrm{kg} \mathrm{KG} / \mathrm{Tag}$ i.v. $)[15,16,19]$.

Rifampicin wirkt in Kombination mit Isoxazolylpenicillin prinzipiell antagonistisch. Dennoch ist die zusätzliche Behandlung mit Rifampicin ratsam bei

- Nachweis von Abszessen,

- intrakardialen Fisteln oder

- polymerassoziierter infektiöser Endokarditis.
Dies liegt daran, dass Rifampicin auch auf phagozytierte Staphylokokken wirkt und in vitro die Sterilisierung von Abszessen beschleunigt.

Gramnegative Erreger und Pilze | Endokarditiden durch gramnegative Bakterien und Pilze machen in Zentraleuropa weniger als $10 \%$ aller IE aus. Standardisierte Empfehlungen zur Behandlung sind nicht sinnvoll, da die Sensibilität gegenüber Antibiotika/Antimykotika stark differiert. Die Therapiestrategie ist von der Empfindlichkeitsprüfung in vitro abhängig und sollte mit einem Kompetenzzentrum abgesprochen werden. 
Kulturnegative Endokarditiden | Die Therapie berücksichtigt die klinische Symptomatik: Bei subakutem Beginn richtet sich die Behandlung primär gegen penicillinempfindliche Streptokokken. Bei akuten Verläufen empfiehlt sich die Kombinationsbehandlung mit Vancomycin und Gentamicin, die auch gegen oxacillin- und methicillinresistente Staphylokokken wirksam und bei Penicillinallergien einsetzbar ist. Kommen gram-negative Bakterien in Betracht, wird zusätzlich Ciprofloxacin empfohlen.

\section{Management typischer Komplikationen}

Wenn typische Komplikationen auftreten, verschlechtert sich die Prognose der akuten IE.

Im Fall einer Komplikation ist individuell zu prüfen, ob zusätzlich zur medikamentösen Therapie eine dringliche chirurgische Intervention indiziert ist.

Sowohl mechanische als auch biologische Herzklappenprothesen sind geeignet - gelegentlich können auch Klappenrekonstruktionen erfolgen. Bei besonders ausgedehnten Infektionen werden Homografts oder klappentragende Conduits eingesetzt. Bei zahlreichen Komplikationen führt die chirurgische Intervention zu einer deutlichen Prognoseverbesserung [2, 3, 5, 20-22] .

Akute Klappeninsuffizienzen | Tritt im Gefolge einer Endokarditis eine akute Klappeninsuffizienz auf, ist die Prognose besonders schlecht: Das Myokard ist nicht an die akute Volumenbelastung adaptiert. Die ansteigenden Wandspannungsparameter führen schnell zur Erschöpfung der myokardialen Adaptationsmechanismen - insbesondere bei akuter Aortenklappeninsuffizienz gibt es kaum Möglichkeiten einer medikamentösen Intervention. Bei schwerer Aortenklappeninsuffizienz ist eine Herzfrequenz von 120-125/ min optimal, um die Diastolendauer zu verkürzen und die Regurgitationsfraktion zu senken. Wird diese Frequenz spontan deutlich verfehlt - z. B. wegen entzündlich bedingter AV-Blockierungen - ist die passagere Schrittmachertherapie sinnvoll. Negativ-chronotrope und bradykardisierende Medikamente sind kontraindiziert. Dobutamin kann kurzfristig zum Anstieg des Herzindex genutzt werden [5].

Mitrale Abklatschvegetationen I Abklatschvegetationen einer primären Aortenklappen-IE auf das anteriore Mitralsegel, sog. „Kissing“ Vegetation, sind mit der Gefahr verbunden, dass auch diese Klappe zerstört wird. In der Mehrzahl der Fälle ist es möglich, die Klappe mit einer frühzeitigen Operation zu erhalten [23].

Systemische Thromboembolien | Das Risiko einer Thromboembolie ist bei einer Mitralklappenen- a Kurzinfusion über 30 min

b bevorzugt bei Patienten $>65$ Jahre oder eingeschränkter Nierenfuntion

c Bei unkompliziertem Erkrankungsverlauf und kurzer Erkrankungsdauer ( $<3$ Monate) kann nur bei Nativklappen-IE die Therapiedauer insgesamt auf 2 Wochen reduziert werden, wenn Gentamicin zusätzlich gegeben wird.

${ }^{d}$ Kurzinfusion über 30 min nach Applikation des $\beta$-Laktam-Antibiotikums. Serumspiegelkontrollen erforderlich: Gentamicinspiegel $<1 \mathrm{mg} / \mathrm{L}$, maximale Tagesgesamtdosis für Gentamicin $240 \mathrm{mg}$, Serumspitzenspiegel für Tobramycin $>12 \mathrm{mg} / \mathrm{L}$

e bei empfindlichen Erregern alternativ Netilmicin (1-mal 4-5 mg/ kg/24h)

f Kurzinfusion über mindestens 60 min; liegt der Vancomycinserumspiegel unter $25 \mathrm{mg} / \mathrm{ml}$, kann die ED erhöht werden; maximale Tagesgesamtdosis $2 \mathrm{~g}$, Vancomycintalspiegel $15-20 \mathrm{mg} / \mathrm{L}$

g der klinische Benefit von Gentamicin konnte nicht sicher gezeigt werden, daher optional bei Nativ-IE, empfohlen bei Prothesen-IE

h identische Therapiedauer für die Einzelkomponenten einer kombinierten Antibiotikatherapie, da nur die Kombination mit dem Aminoglykosid bakterizid wirksam ist; bei komplizierten Verläufen, echokardiografischem Nachweis großer Vegetationen (>5 mm), einer mehr als 3-monatigen Erkrankungsdauer und Prothesen-IE ist eine 6-wöchige Therapie vorzuziehen

' bei „high-level“ Gentamicin-Resistenz (MHK > $500 \mathrm{mg} / \mathrm{L}$ ) sofern empfindlich durch Streptomycin $15 \mathrm{mg} / \mathrm{kg} 24 \mathrm{~h}$ in Einzeldosen (I A) oder verlängerte BetalactamTherapie ersetzen; alternativ Ampicillin plus Ceftriaxon bei E. faecalis (Ila B) bei Betalactam-Resistenz: durch Betalactamase-Ampicillin-Sulbactam (I C), durch PBP5-Alteration Vancomycin-Schema bei Multiresistenz gegen Aminoglykoside, Betalactam-Therapie und Vancomycin wird alternativ empfohlen: Daptomycin $6 \mathrm{mg} / \mathrm{kg} / 24 \mathrm{~h}$ (ggf. 8-12 mg/ kg) als Einmaldosis, Linezolid 2-mal $600 \mathrm{mg} / 24 \mathrm{~h}$ (cave: hämatologische Toxizität), Quinupristin-Dalfopristin 3-mal 7,5 mg/kg/24h oder Imipinen 3-4-mal $1 \mathrm{~g} / 24 \mathrm{~h}$ plus Ampicillin oder Ceftriaxon plus Ampicillin jeweils 8 Wochen

k Lediglich bei unkomplizierten Erkrankungsverläufen ist eine nur 4-wöchige Therapiedauer vertretbar.

I Mehr als die Hälfte der koagulasenegativen Staphylokokken sind Oxacillin-resistent.

${ }^{m}$ bei Koagulase-negativen Staphylokokken und gezielter Indikation (Abszesse, intrakardiale Fisteln, Implantation prothetischen Materials) zusätzlich 2-mal $600 \mathrm{mg}$ Rifampicin während der gesamten Therapiedauer

n überwiegend Ampicillin-resistent

- 6 Wochen bei Prothesenendokarditis

P bei intermediärer Vancomycin-Resistenz (MHKVanco 4-16 mg/L) oder „high-level“ Vancomycin-Resistenz wird empfohlen: Daptomycin 1-mal $6 \mathrm{mg} / \mathrm{kg} / 24 \mathrm{~h}$ (ggf. $8-12 \mathrm{mg} / \mathrm{kg}$ ), Quinopristin-Dalfopristin (Synerid) mit/ ohne Betalactam-Antibiotikum, Betalactam-Antibiotikum plus Oxazolidinone oder plus Vancomycin

Erläuterung der Fußnoten zu Tab. 1.

dokarditis höher als bei einer isolierten Aortenklappenendokarditis und in gewissem Umfang abhängig vom Erreger: Bei Streptokokken ist die Inzidenz geringer als bei Staphylokokken und Enterokokken $[1,3]$. Die TTE unterschätzt im Vergleich zur TEE die Vegetationsgröße regelhaft [9, 20]. Große Vegetationen neigen eher zu thromboembolischen Komplikationen als solche, die kleiner als $10 \mathrm{~mm}$ sind. Dies gilt insbesondere für Vegetationen, die an den sehr mobilen Segelanteilen der Mitralklappe haften und deshalb hohen Beschleunigungen ausgesetzt sind.

Nach erstmaliger Thromboembolie ist das Rezidivrisiko sowohl bei Mitral- als auch bei Aortenklappen-IE beträchtlich, wenn nach Komplikationseintritt mit TEE weiterhin flottierende Vegetationen oder spontaner Echokontrast nachweisbar 


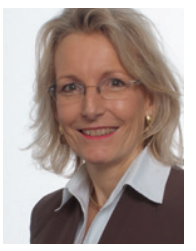

Prof. Dr. med. Cornelia Piper ist stellvertr. Direktorin der Klinik für Kardiologie des Herz- und Diabeteszentrum NRW der Ruhr-Universität Bochum.

cpiper@hdz-nrw.de

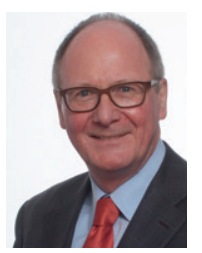

Univ.-Prof. Dr. med. Dieter Horstkotte

ist Direktor der Klinik für Kardiologie und stellvertretender ärztlicher Direktor des Herz- und Diabeteszentrum NRW der Ruhr-Universität Bochum.

akleemeyer@hdz-nrw.de

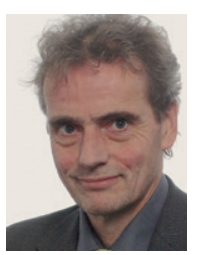

Prof. Dr. med. Lothar Faber ist Oberarzt der Klinik für Kardiologie und Leiter des Echolabors am Herz- und Diabeteszentrum NRW der Ruhr-Universität Bochum. Ifaber@hdz-nrw.de

\section{Interessenkonflikt}

Die Autoren geben an, dass kein Interessenkonflikt besteht.

DOI 10.1055/s-0041-100499 VNR 2760512015147121764 Dtsch Med Wochenschr 2015; 140: 353-362

(C) Georg Thieme Verlag KG . Stuttgart · New York ·

ISSN 0012-0472 sind $[1,2,3,5]$. In mehr als der Hälfte dieser Fälle tritt innerhalb von 30 Tagen ein Embolierezidiv auf.

Eine effektive Antikoagulation vermindert das Embolierisiko bei florider Endokarditis nicht, erhöht aber das Risiko für Blutungen erheblich - insbesondere für zerebrale Blutungen. Die Antikoagulationsbehandlung ist deshalb bei allen Patienten obsolet, die nicht aus anderen Gründen (mechanischer Herzklappenersatz, Vorhofflimmern, etc.) eine Indikation dazu haben [3].

ZNS-Beteiligung bei IE I Sowohl bei Nativklappenals auch bei Prothesenendokarditiden treten in etwa einem Drittel der Krankheitsverläufe neurologische Komplikationen auf. Dazu gehören z.B.:

- intrakranielle, okkludierende Embolien

- zerebrale Blutungen bei mykotischem Aneurysma

- Hirnabszess

- Meningitis

- Meningoenzephalitis

Die Mortalität der IE mit ZNS-Beteiligung beträgt $41 \%$, ohne neurologische Komplikationen dagegen nur $15 \%$ [24].

Nach zerebralen Embolien ist bei fortbestehendem Thromboembolierisiko möglichst innerhalb der ersten $48 \mathrm{~h}$ zu operieren. Die nach Ablauf von etwa $72 \mathrm{~h}$ progrediente Störung der Blut-HirnSchranke verschlechtert die Prognose. Sie ist indirekt auch dafür verantwortlich, dass eine Operation mehr als 8 Tage nach dem Ereignis - im Vergleich zur konservativen Behandlung - nicht mehr zu einer Prognoseverbesserung führt. Bei späten Operationen nimmt die Rate sekundärer zerebraler Blutungen stark zu. Vor der Operation ist ein CCT zum Ausschluss einer zerebralen Reperfusionsblutung zwingend [2, 3, 25]

Besonderheiten der Kunstklappenendokarditis I Da die Infektionen ihren Ausgang vom Nahtring oder von nahtringnahen Thromben nehmen, sind periprothetische Dehiszenzen und Abszesse häufig. Prothesenendokarditiden, die durch koagulasenegative Staphylokokken oder Enterokokken verursacht werden, sind schwierig zu sanieren. Trotz höchstdosierter Kombinationstherapien synergistisch oder additiv wirksamer Antibiotika gelingt dies selten. Patienten mit Kunstklappen-IE sind zusätzlich mit Rifampicin zu behandeln, da Rifampicin aktiv in Granulozyten angereichert wird und die Sterilisation von Abszessen beschleunigt. In den meisten Fällen empfiehlt sich ein primär kombiniertes, chirurgisches und antimikrobielles Management. Die antibiotische Kombinationstherapie ist in diesen Fällen nach dem operativen Herzklappenersatz intravenös für 6 Wochen fortzusetzen $[2,3,5,22]$.

\section{Primär chirurgische Therapie}

Bei folgenden Befunden bzw. Komplikationen empfiehlt sich ein primär kombiniertes chirurgisches und antimikrobielles Management [22]:

- progrediente therapierefraktäre Herzinsuffizienz bei schwerer Aortenklappeninsuffizienz

- progrediente therapierefraktäre Herzinsuffizienz bei schwerer Mitralklappeninsuffizienz

- persistierende Sepsis über $>48 \mathrm{~h}$ trotz adäquater antibiotischer Therapie

- nach Embolie weiterhin Nachweis einer großen $(>10 \mathrm{~mm}$ ) flottierenden Vegetation

- große flottierende Vegetation (>10 mm an der Mitralklappe, >15-20 mm an der Aortenklappe)

- Abklatschvegetation an der Mitralklappe bei primärer Aortenklappen-IE

- akutes Nierenversagen

- Polymerinfektion (Kunstklappenprothesen, Katheter, Schrittmacherkabel) durch Staphylokokken oder resistente Enterokokken

- Kunstklappenprothesen-IE mit paraprothetischen Abszessen

- Infektionen mit antimikrobiell schwer zu behandelnden oder resistenten Mikroorganismen, inkl. Pilze

Fazit

- Voraussetzungen für die Entstehung einer infektiösen Endokarditis (IE) ist die Besiedlung des Endokards durch vermehrungsfähige Mikroorganismen im Zuge einer Bakteriämie.

- Die IE wird zu $90 \%$ von grampositiven Kokken (Streptokokken, Staphylokokken, Enterokokken) verursacht, wobei eine Staphylokokken-IE häufig einen foudroyanten Verlauf nimmt.

- Bei IE-Verdacht ist immer eine transthorakale und meist eine transösophageale Echokardiografie unverzüglich erforderlich; auch müssen mind. 3 Blutkultursets unabhängig vom Fieberverlauf entnommen werden.

- Die antibiogrammgerechte antibiotische Therapie erfolgt in aller Regel als intravenöse Kombinationstherapie über 4-6 Wochen.

- Treten typische Komplikationen auf, ist meist eine dringliche chirurgische Intervention indiziert.

- Polymerassoziierte IE (Kunstklappe, Elektrodenkabel etc.) sind komplikationsträchtig und erfordern meist die komplette Entfernung des gesamten Polymermaterials.

\section{Literatur}

1 Horstkotte D. Mikrobielle verursachte Endokarditis: Klinische und tierexperimentelle Untersuchungen. Darmstadt: Steinkopff; 1995

2 Murdoch DR, Corey GR, Hoen B et al. Clinical presentation, etiology, and outcome of infective endocarditis in the 21st century: the International Collaboration on Endocarditis-Prospective Cohort Study. Arch Intern Med 2009; 169: 463-473

Vollständiges Literaturverzeichnis unter http://dx.doi.org/10.1055/s-0041-100499 
3 Habib G, Hoen B, Tornos P et al. Guidelines on the prevention, diagnosis, and treatment of infective endocarditis (new version 2009): the Task Force on the Prevention, Diagnosis, and Treatment of Infective Endocarditis of the European Society of Cardiology (ESC). Endorsed by the European Society of Clinical Microbiology and Infectious Diseases (ESCMID) and the International Society of Chemotherapy (ISC) for Infection and Cancer. Eur Heart ] 2009; 30: 2369-2413

4 Horstkotte D, Weist K, Rüden H. Better understanding of the pathogenesis of prosthetic valve endocarditis-recent perspectives for prevention strategies. J Heart Valve Dis 1998; 7: 313-315

5 Horstkotte D, Wagener J, Piper C. Endokarditis. In: Paumgartner G, Hrsg. Therapie Innerer Krankheiten. Berlin: Springer, 2005; 137-153

6 Kreuzpaintner G, Horstkotte D, Heyll A et al. Increased risk of bacterial endocarditis in inflammatory bowel disease. Am J Med 1992; 92: 391-395

7 Evangelista A, Gonzalez-Alujas MT. Echocardiography in infective endocarditis. Heart 2004; 90: 614-617

8 Cormier B, Vitoux B, Starkman C et al. Value of transesophageal echocardiography. From a preliminary experience of 532 cases. Arch Mal Coeur Vaiss 1990; 83: 23-29

9 Rasmussen RV, Hoest U, Arpi M et al. Prevalence of infective endocarditis in patients with Staphylococcus aureus bacteraemia: the value of screening with echocardiography. Eur ] Echocardiogr 2011;12: 414-420

10 Erbel R, Rohmann S, Drexler M et al. Improved diagnostic value of echocardiography in patients with infective endocarditis by transoesophageal approach. A prospective study. Eur Heart J 1988; 9: 43-53

11 Durack DT, Lukes AS, Bright DK. New criteria for diagnosis of infective endocarditis: utilization of specific echocardiographic findings. Duke Endocarditis Service. Am J Med 1994; 96: 200-209

12 Vollmer T, Piper C, Horstkotte D et al. 23S rDNA real-time polymerase chain reaction of heart valves: a decisive tool in the diagnosis of infective endocarditis. Eur Heart ] 2010; 31: 1105-1113

13 Burnie JP, Clark I. Diagnosing endocarditis with the cloned $112 \mathrm{kDA}$ antigen of Enterococcus faecalis. J Immunol Methods 1989; 123: 217-225

14 Raoult D, Casalta JP, Richet $\mathrm{H}$ et al. Contribution of systematic serological testing in diagnosis of infective endocarditis. J Clin Microbiol 2005; 43: 5238-5242
15 Levine DP, Lamp KC. Daptomycin in the treatment of patients with infective endocarditis: experience from a registry. Am J Med 2007; 120: S28-33

16 Kullar R, Davis SL, Levine DP et al. High-dose daptomycin for treatment of complicated gram-positive infections: a large, multicenter, retrospective study. Pharmacotherapy 2011; 31: 527-536

17 Jacqueline C, Navas D, Batard E et al. In vitro and in vivo synergistic activities of linezolid combined with subinhibitory concentrations of imipenem against methicillin-resistant Staphylococcus aureus. Antimicrob Agents Chemother 2005; 49: 45-51

18 Perichon B, Courvalin P. Synergism between betalactams and glycopeptides against VanA-type methicillin-resistant Staphylococcus aureus and heterologous expression of the vanA operon. Antimicrob Agents Chemother 2006; 50: 3622-3630

19 Fowler VG, Jr., Boucher HW, Corey GR et al. Daptomycin versus standard therapy for bacteremia and endocarditis caused by Staphylococcus aureus. N Engl J Med 2006; 355: 653-665

20 Mügge A, Daniel WG, Frank G et al. Echocardiography in infective endocarditis: reassessment of prognostic implications of vegetation size determined by the transthoracic and the transesophageal approach. J Am Coll Cardiol 1989; 14: 631-638

21 Horstkotte D, Bircks W, Loogen F. Infective endocarditis of native and prosthetic valves - the case for prompt surgical intervention? A retrospective analysis of factors affecting survival. Z Kardiol 1986; 75 Suppl 2: 168-182

22 Mirabel M, Sonneville R, Hajage D et al. Long-term outcomes and cardiac surgery in critically ill patients with infective endocarditis. Eur Heart J 2014; 35 : 1195-1204

23 Piper C, Hetzer R, Körfer R et al. The importance of secondary mitral valve involvement in primary aortic valve endocarditis; the mitral kissing vegetation. Eur Heart J 2002; 23: 79-86

24 Horstkotte D, Piper C, Wiemer M. ZNS-Beteiligung bei akuter Endokarditis. In: Prange H, Bitsch A, Hrsg. Bakterielle ZNS-Erkrankungen bei systemischen Infektionen. Darmstadt: Steinkopff, 1997; 45-63

25 Piper C, Wiemer M, Schulte HD et al. Stroke is not a contraindication for urgent valve replacement in acute infective endocarditis. J Heart Valve Dis 2001; 10: 703-711
Der Beitrag wurde am 26.06.2015 gemäß folgendem Erratum korrigiert:

Im Beitrag „Aortenklappenendokarditis: Diagnostik und Therapie“ (Dtsch Med Wochenschr 2015; 140: 353-362) muss es auf S. 358, Tab. 1 richtig heißen: „Oxacillin-empfindliche Staphylokokken (MSSA): $\mathrm{MHK}_{\text {Oxa }}<1 \mathrm{mg} / \mathrm{L}^{\prime \prime}$. 


\section{CME-Fragen}

CME-Teilnahme

- Viel Erfolg bei Ihrer CME-Teilnahme unter http://cme.thieme.de

- Diese Fortbildungseinheit ist 12 Monate online für eine CME-Teilnahme verfügbar.

- Sollten Sie Fragen zur Online-Teilnahme haben, unter http:// cme.thieme.de/hilfe finden Sie eine ausführliche Anleitung.

1. Welche Erreger können keine infektiöse Endokarditis verursachen?

a. Candida albicans

b. Staphylococcus aureus

c. E. coli

d. Chlamydien

e. Adenoviren

2. Das Risiko für einen Patienten mit einem angeborenen oder erworbenen Herzklappenfehler, an einer Endokarditis zu erkranken, wird nicht begünstigt durch

a. terminale Niereninsuffizienz

b. Diabetes mellitus

c. arterielle Hypertonie

d. immunsuppressive Therapie

e. Alkoholabusus

3. Bei welchem Endokarditiserreger finden sich gehäuft gastrointestinale Tumore?

a. Streptococcus bovis

b. Chlamydien

c. Candida albicans

d. Staphylococcus aureus

e. Staphylococcus epidermidis

4. Welches dieser Symptome legt einen dringenden Verdacht auf eine Endokarditis nahe?

a. Leistungsknick

b. Übelkeit und Erbrechen

c. Abgeschlagenheit und Mattigkeit

d. Fieber plus Haut- und Retinamanifestationen

e. anhaltende Kopfschmerzen

5. Welche Aussage bezüglich der Entnahme von Blutkulturen (BK) bei Verdacht auf Endokarditis ist korrekt?

a. BK sollten während einer Fieberspitze abgenommen werden.

b. BK sollten in einem Brutschrank aufbewahrt werden.

c. Es sollte arterielles Blut entnommen werden.

d. Nur selten sind Endokarditiden kulturnegativ

e. Leitliniengerecht sollten mindestens 3 Blutkultursets abgenommen werden.

6. Wie lange wird ein Patient mit nativer Aortenklappen-IE mit blutkulturell nachgewiesenen oxazillinempfindlichen $\left(\mathrm{MHK}_{\mathrm{Oxa}}<0,1 \mathrm{mg} / \mathrm{L}\right)$ Staphylokokken und einer Vegetationsgröße von $8 \mathrm{~mm}$ in aller Regel intravenös behandelt?

a. 1 Woche

b. 2 Wochen

c. 3 Wochen

d. 4 Wochen

e. 8 Wochen

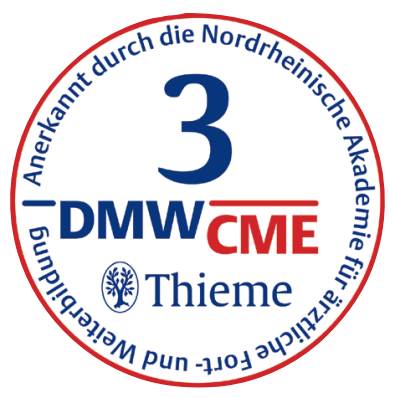

7. Mit welcher antibiotischen Kombinationstherapie wird ein Patient mit penicillinempfindlicher ( $\mathrm{MHK}_{\mathrm{Pen}} 4 \mathrm{mg} / \mathrm{L}$ ) Enterococcus faecalis-Aortenklappen-IE über 4-6 Wochen behandelt?

a. Metronidazol plus Gentamicin

b. Ampicillin oder Penicillin G plus Gentamicin

c. Imipenem plus Metronidazol

d. Avalox plus Ciprobay

e. Avalox plus Gentamicin

8. Wie nennt man die Abklatschvegetation am vorderen Mitralklappensegel bei Aortenklappen-IE?

a. „Kissing“ Vegetation

b. „Ablagerung“

c. „Smile“

d. Prolaps

e. Flail

9. Welche Aussage zur antibiotischen Therapie der Endokarditis ist richtig?

a. Das Monitoring der Wirkspiegel von Aminoglykosiden und Vancomycin sollte engmaschig durchgeführt werden, um eine Akkumulation der nephrotoxischen Substanzen zu verhindern.

b. Auch kritisch kranke Patienten dürfen nur antibiotisch therapiert werden, wenn zuvor der Keim eindeutig identifiziert wurde.

c. Die Antibiotikatherapie ist immer mit einem Antimykotikum zu kombinieren.

d. Die Antibiotika werden bei IE oral verabreicht.

e. „High Level Resistance (HLR)“ gegen Gentamicin gibt es bei Enterococcus-Stämmen nicht.

10. Welche Aussage zu Komplikationen einer Aortenklappenendokarditis ist korrekt?

a. Zu chirurgischen Intervention sind nur mechanische Herzklappen geeignet.

b. Bei Staphylokokken ist die Inzidenz von Thromboembolien geringer als bei Streptokokken.

c. Bei schwerer Aortenklappeninsuffizienz ist eine Herzfrequenz von 120-125/min optimal.

d. Eine Antikoagulation erhöht zwar das Risiko für Blutungen, senkt aber gleichzeitig das Embolierisiko bei florider Endokarditis.

e. Nach operativem Herzklappenersatz muss man die antibiotische Kombinationstherapie intravenös für 2 Wochen fortsetzen. 


\section{Angaben zur Person}

Name, Vorname, Titel:

Straße, Hausnr:

PLZ, Ort:

Anschrift: $\square$ privat $\square$ dienstlich

Ich bin Mitglied der Ärztekammer (bitte Namen der Kammer eintragen):

Jahr meiner Approbation:

Ich befinde mich in der Weiterbildung zum:

Ich habe eine abgeschlossene Weiterbildung in ... (bitte Fach eintragen):

Ich bin tätig als: $\square$ Assistenzarzt $\square$ Oberarzt $\square$ Chefarzt $\square$ niedergelassener Arzt $\square$ Sonstiges Ich bin DMW-Abonnent: $\square$ ja $\square$ nein Falls nein: ich habe den Fragebogen aus / von: $\square$ Thieme-connect $\square$ Kollegen $\square$ der Klinik $\square$ einer Bibliothek $\square$ Sonstiges

Lernerfolgskontrolle (Eine Antwort pro Frage ankreuzen)
1. $\square$ A $\square$ B $\square$ C $\square$ D $\square$ E
2. $\square \mathrm{A} \quad \square \mathrm{B} \quad \square \mathrm{C} \quad \square \mathrm{D} \quad \square \mathrm{E}$
3. $\square \mathrm{A} \quad \square \mathrm{B} \quad \square \mathrm{C} \quad \square \mathrm{D} \quad \square \mathrm{E}$
4. $\square$ A $\square$ B $\square$ C $\square$ D $\square$ E
5. $\square \mathrm{A} \quad \square \mathrm{B} \quad \square \mathrm{C} \quad \square \mathrm{D} \quad \square \mathrm{E}$
6. $\square \mathrm{A} \quad \square \mathrm{B} \quad \square \mathrm{C} \quad \square \mathrm{D} \quad \square \mathrm{E}$
7. $\square$ A $\square$ B $\square$ C $\square$ D $\quad \square$ E
8. $\square$ A $\square$ B $\square$ C $\square$ D $\square$ E
9. $\square \mathrm{A} \square \mathrm{B} \quad \square \mathrm{C} \quad \square \mathrm{D} \quad \square \mathrm{E}$
10. $\square \mathrm{A} \quad \square \mathrm{B} \quad \square \mathrm{C} \quad \square \mathrm{D} \quad \square \mathrm{E}$

Ich versichere, dass ich die Beantwortung der Fragen selbst und ohne fremde Hilfe durchgeführt habe

Ort, Datum:

Unterschrift:

Bitte in dieses Feld Ihre DMW Abonnement-Nummer eintragen:

\section{Fragen zur Zertifizierung}

1. Das Thema des Beitrages kommt in meiner ärztlichen Tätigkeit $\square$ häufig vor $\square$ selten vor $\square$ regelmäßig vor $\square$ gar nicht vor

2. Bei diesem Thema habe ich $\square$ eine feste Gesamtstrategie $\square$ keine Strategie $\square$ noch offene Einzelprobleme

3. In Bezug auf das Thema des Beitrages

$\square$ fühle ich mich nach dem Studium des Beitrags in meiner Strategie bestätigt

$\square$ habe ich meine Strategie verändert:

$\square$ habe ich erstmals eine einheitliche Strategie erarbeitet

$\square$ habe ich keine einheitliche Strategie ableiten können

4. Wurden aus der Sicht Ihrer täglichen Praxis heraus wichtige Aspekte des Themas

nicht erwähnt: $\square$ ja, welche $\square$ nein

zu knapp abgehandelt? $\square$ ja, welche $\square$ nein

überbewertet? $\square$ ja, welche $\square$ nein

5. Verständlichkeit des Beitrages

$\square$ Der Beitrag ist nur für Spezialisten verständlich

$\square$ Der Beitrag ist auch für Nicht-Spezialisten verständlich

6. Beantwortung der Fragen

$\square$ Die Fragen lassen sich aus dem Studium des Beitrags allein beantworten

$\square$ Die Fragen lassen sich nur unter Zuhilfenahme zusätzlicher Literatur beantworten

7. Die Aussagen des Beitrages benötigen eine ausführlichere Darstellung

$\square$ zusätzlicher Daten

$\square$ von Befunden bildgebender Verfahren

$\square$ die Darstellung ist ausreichend

8. Wieviel Zeit haben Sie für das Lesen des Beitrages und der Bearbeitung des Quiz benötigt?

Zertifizierungsfeld (wird durch die DMW ausgefülltt)

Ihr Ergebnis:

Sie haben

von 10 Fragen richtig beantwortet.

Sie haben $\square$ bestanden und 3 Punkte erworben

$\square$ nicht bestanden $\square$ ungültig,weil:

Stuttgart, den Stempel/Unterschrift 